Pacific

Journal of

Mathematics

\title{
TILINGS DEFINED BY AFFINE WEYL GROUPS
}

ECKHARD MEINRENKEN

Volume $242 \quad$ No. 2

October 2009 


\title{
TILINGS DEFINED BY AFFINE WEYL GROUPS
}

\author{
ECKHARD MEINRENKEN
}

\begin{abstract}
Let $W$ be a Weyl group, presented as a reflection group on a Euclidean vector space $V$, and $C \subset V$ an open Weyl chamber. In a recent paper, Waldspurger proved that the images $(\mathrm{id}-w)(C)$ for $w \in W$ are all disjoint, with union the closed cone spanned by the positive roots. We prove that similarly, the images $(\mathrm{id}-w)(A)$ of the open Weyl alcove $A$, for $w \in W^{\text {aff }}$ in the affine Weyl group, are disjoint and their union is $V$.
\end{abstract}

\section{Introduction}

Let $W$ be the Weyl group of a simple Lie algebra, presented as a crystallographic reflection group in a finite-dimensional Euclidean vector space $(V,\langle\cdot, \cdot\rangle)$. Choose a fundamental Weyl chamber $C \subset V$, and let $D$ be its dual cone, that is, the open cone spanned by the corresponding positive roots. Waldspurger [2007] proved the following remarkable result. Consider the linear transformations (id $-w): V \rightarrow V$ defined by elements $w \in W$.

Theorem 1.1 (Waldspurger). The images $D_{w}:=(\mathrm{id}-w)(C)$ for $w \in W$ are all disjoint, and their union is the closed cone spanned by the positive roots:

$$
\bar{D}=\bigcup_{w \in W} D_{w} .
$$

For instance, the identity transformation $w=$ id corresponds to $D_{\text {id }}=\{0\}$ in this decomposition, while the reflection $s_{\alpha}$ defined by a positive root $\alpha$ corresponds to the open half-line $D_{s_{\alpha}}=\mathbb{R}_{>0} \cdot \alpha$.

The aim of this note is to prove a similar result for the affine Weyl group $W^{\text {aff }}$. Recall that $W^{\text {aff }}=\Lambda \rtimes W$, where the coroot lattice $\Lambda \subset V$ acts by translations. Let $A \subset C$ be the Weyl alcove, with $0 \in \bar{A}$.

Theorem 1.2. The images $V_{w}=(\mathrm{id}-w)(A)$ for $w \in W^{\text {aff }}$ are all disjoint, and their union is $V$ :

$$
V=\bigcup_{w \in W^{\text {aff }}} V_{w}
$$

MSC2000: 20F55, 22E46.

Keywords: affine Weyl group, reflection groups, alcoves.

The author is partially supported by an NSERC Discovery Grant and a Steacie Fellowship. 


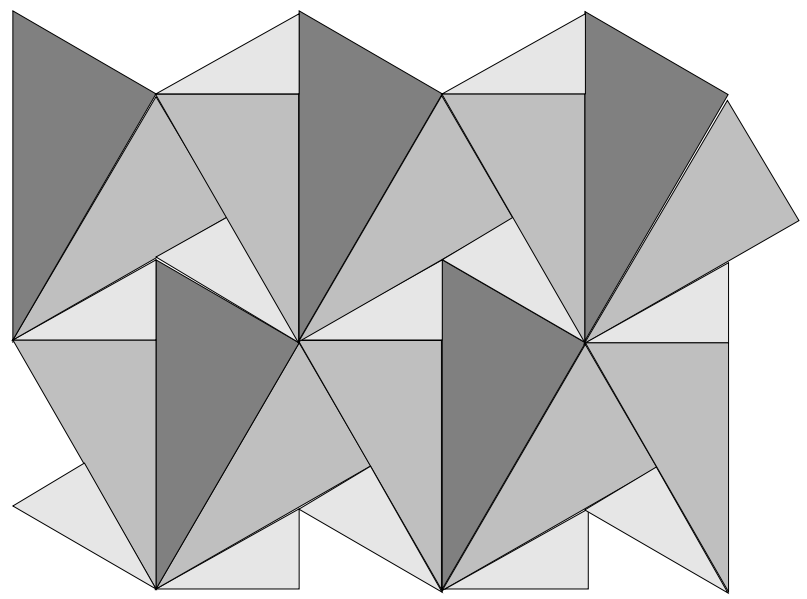

The figure above is a picture of the resulting tiling of $V$ for the root system $\mathbf{G}_{2}$. Up to translation by elements of the lattice $\Lambda$, there are five 2-dimensional tiles, corresponding to the five Weyl group elements with trivial fixed point set. With $s_{1}$ and $s_{2}$ denoting the simple reflections, the lightly shaded polytopes are labeled by the Coxeter elements $s_{1} s_{2}$ and $s_{2} s_{1}$, the medium shaded polytopes by $\left(s_{1} s_{2}\right)^{2}$ and $\left(s_{2} s_{1}\right)^{2}$, and the darkly shaded polytope by the longest Weyl group element $w_{0}=\left(s_{1} s_{2}\right)^{3}$.

One also has the following related statement.

Theorem 1.3. Suppose $S \in \operatorname{End}(V)$ with $\|S\|<1$. Then the sets $V_{w}^{(S)}=(S-w)(A)$ for $w \in W^{\text {aff }}$ are all disjoint, and their closures cover $V$ :

$$
V=\bigcup_{w \in W^{\text {aff }}} \bar{V}_{w}^{(S)}
$$

Note that for $S=0$ the resulting decomposition of $V$ is just the Stiefel diagram, while for $S=\tau$ id with $\tau \rightarrow 1$ one recovers the decomposition from Theorem 1.2.

The proof of Theorem 1.2 is in large parts parallel to Waldspurger's [2007] proof of Theorem 1.1. We will nevertheless give full details so the paper is self-contained.

\section{Notation}

With no loss of generality we will take $W$ to be irreducible. Let $\Re \subset V$ be the set of roots, $\left\{\alpha_{1}, \ldots, \alpha_{l}\right\} \subset \mathfrak{R}$ a set of simple roots, and

$$
C=\left\{x \mid\left\langle\alpha_{i}, x\right\rangle>0, i=1, \ldots, l\right\}
$$

the corresponding Weyl chamber. We denote by $\alpha_{\max } \in \mathfrak{R}$ the highest root, and $\alpha_{0}=-\alpha_{\max }$ the lowest root. The open Weyl alcove is the $l$-dimensional simplex 
defined as

$$
A=\left\{x \mid\left\langle\alpha_{i}, x\right\rangle+\delta_{i, 0}>0, i=0, \ldots, l\right\} .
$$

Its faces are indexed by the proper subsets $I \subset\{0, \ldots, l\}$, where $A_{I}$ is given by inequalities $\left\langle\alpha_{i}, x\right\rangle+\delta_{i, 0}>0$ for $i \notin I$ and equalities $\left\langle\alpha_{i}, x\right\rangle+\delta_{i, 0}=0$ for $i \in I$. Each $A_{I}$ has codimension $|I|$. In particular, $A_{i}=A_{\{i\}}$ are the codimension 1 faces, with $\alpha_{i}$ as inward-pointing normal vectors. Let $s_{i}$ be the affine reflections across the affine hyperplanes supporting $A_{i}$, that is,

$$
s_{i}: x \mapsto x-\left(\left\langle\alpha_{i}, x\right\rangle+\delta_{i, 0}\right) \alpha_{i}^{\vee} \text { for } i=0, \ldots, l,
$$

where $\alpha_{i}^{\vee}=2 \alpha_{i} /\left\langle\alpha_{i}, \alpha_{i}\right\rangle$ is the simple coroot corresponding to $\alpha_{i}$. The Weyl group $W$ is generated by the reflections $s_{1}, \ldots, s_{l}$, while the affine Weyl group $W^{\text {aff }}$ is generated by the affine reflections $s_{0}, \ldots, s_{l}$. The affine Weyl group is a semidirect product

$$
W^{\mathrm{aff}}=\Lambda \rtimes W,
$$

where the coroot lattice $\Lambda=\mathbb{Z}\left[\alpha_{1}^{\vee}, \ldots, \alpha_{l}^{\vee}\right] \subset V$ acts on $V$ by translations. For any $w \in W^{\text {aff }}$, we will denote by $\tilde{w} \in W$ its image under the quotient map $W^{\text {aff }} \rightarrow W$, that is, $\tilde{w}(x)=w(x)-w(0)$, and by $\lambda_{w}=w(0) \in \Lambda$ the corresponding lattice vector.

The stabilizer of any element of $A_{I}$ is the subgroup $W_{I}^{\text {aff }}$ generated by $s_{i}$ for $i \in I$. It is a finite subgroup of $W^{\text {aff }}$, and the map $w \mapsto \tilde{w}$ induces an isomorphism onto the subgroup $W_{I}$ generated by $\tilde{s}_{i}$ for $i \in I$. Recall that $W_{I}$ is itself a Weyl group (not necessarily irreducible): Its Dynkin diagram is obtained from the extended Dynkin diagram of the root system $\mathfrak{R}$ by removing all vertices that are in $I$.

\section{The top-dimensional polytopes}

For any $w \in W^{\text {aff }}$, the subset $V_{w}=(\mathrm{id}-w)(A)$ is the relative interior of a convex polytope in the affine subspace $\operatorname{ran}(\mathrm{id}-w)$. Let

$$
W_{\text {reg }}^{\text {aff }}=\left\{w \in W^{\text {aff }} \mid(\mathrm{id}-w) \text { is invertible }\right\}
$$

and $W_{\text {reg }}=W \cap W_{\text {reg }}^{\text {aff }}$, so that $w \in W_{\text {reg }}^{\text {aff }}$ if and only if $\tilde{w} \in W_{\text {reg. }}$. The top dimensional polytopes $V_{w}$ are those indexed by $w \in W_{\text {reg }}^{\text {aff }}$ and the faces of these polytopes are $V_{w, I}:=(\mathrm{id}-w)\left(A_{I}\right)$. For $w \in W_{\text {reg }}$ and $i=0, \ldots, l$, let

$$
n_{w, i}:=\left(\mathrm{id}-\tilde{w}^{-1}\right)^{-1}\left(\alpha_{i}\right) .
$$

Lemma 3.1. For all $w \in W_{\mathrm{reg}}^{\mathrm{aff}}$, the open polytope $V_{w}$ is given by the inequalities

$$
\left\langle n_{w, i}, \xi+\lambda_{w}\right\rangle+\delta_{i, 0}>0 \quad \text { for } i=0, \ldots, l .
$$

The face $V_{w, I}=(\mathrm{id}-w)\left(A_{I}\right)$ is obtained by replacing the inequalities for $i \in I$ by equalities. 
Proof. For any $\xi=(\mathrm{id}-w) x \in V$, we have

$$
\left\langle\alpha_{i}, x\right\rangle=\left\langle\left(\mathrm{id}-\tilde{w}^{-1}\right)^{-1} \alpha_{i},(\mathrm{id}-\tilde{w}) x\right\rangle=\left\langle n_{w, i},(\mathrm{id}-\tilde{w}) x\right\rangle=\left\langle n_{w, i}, \xi+\lambda_{w}\right\rangle,
$$

since $\tilde{w}^{-1}$ is the transpose of $\tilde{w}$ under the inner product $\langle\cdot, \cdot\rangle$. This gives the description of $V_{w}$ and of its faces $V_{w, I}$.

Lemma 3.2. Suppose $w \in W_{\mathrm{reg}}^{\text {aff }}$ for $i \in\{0, \ldots, l\}$. Then $V_{w, i}=V_{\sigma, i} \subset \operatorname{ran}(\mathrm{id}-\sigma)$ with $\sigma=w s_{i}$. In particular, $\sigma$ is an affine reflection, and $n_{w, i}$ is a vector normal to the affine hyperplane $\mathrm{ran}(\mathrm{id}-\sigma)$. One has $\left\langle n_{w, i}, \alpha_{i}^{\vee}\right\rangle=1$.

Proof. For any orthogonal transformation $g \in \mathrm{O}(V)$ and any reflection $s \in \mathrm{O}(V)$, the dimension of the fixed point set of the orthogonal transformations $g$ and $g s$ differ by \pm 1 . Since $\tilde{w}$ fixes only the origin, it follows that $\tilde{\sigma}$ has a 1-dimensional fixed point set. Hence $\operatorname{ran}(\mathrm{id}-\sigma)$ is an affine hyperplane, and $\sigma$ is the affine reflection across that hyperplane. Since $s_{i}$ fixes $A_{i}$, we have

$$
V_{w, i}=(\mathrm{id}-w)\left(A_{i}\right)=\left(\mathrm{id}-w s_{i}\right)\left(A_{i}\right)=V_{\sigma, i} \subset \operatorname{ran}(\mathrm{id}-\sigma) .
$$

By definition $n_{w, i}-\tilde{w}^{-1} n_{w, i}=\alpha_{i}$. Hence

$$
-2\left\langle n_{w, i}, \alpha_{i}\right\rangle+\left\langle\alpha_{i}, \alpha_{i}\right\rangle=\left\|n_{w, i}-\alpha_{i}\right\|^{2}-\left\|n_{w, i}\right\|^{2}=\left\|\tilde{w}^{-1} n_{w, i}\right\|^{2}-\left\|n_{w, i}\right\|^{2}=0 .
$$

The following proposition indicates how the top-dimensional polytopes $V_{w, i}$ are glued along the polytopes of codimension 1 .

Proposition 3.3. Let $\sigma \in W^{\text {aff }}$ be an affine reflection, that is, $\operatorname{ran}(\mathrm{id}-\sigma)$ is an affine hyperplane. Consider

$$
\xi \in V_{\sigma} \backslash \bigcup_{|I| \geq 2} V_{\sigma, I}
$$

Then there are two distinct indices $i, i^{\prime} \in\{0, \ldots, l\}$ such that $\xi \in V_{\sigma, i} \cap V_{\sigma, i^{\prime}}$. Furthermore, $w=\sigma s_{i}$ and $w^{\prime}=\sigma s_{i^{\prime}}$ are both in $W_{\mathrm{reg}}^{\text {aff }}$, so that $V_{w, i}=V_{\sigma, i}$ and $V_{w^{\prime}, i^{\prime}}=V_{\sigma, i^{\prime}}$, and the polytopes $V_{w}, V_{w^{\prime}}$ are on opposite sides of the affine hyperplane $\operatorname{ran}(\mathrm{id}-\sigma)$.

Proof. Let $n$ be a generator of the 1-dimensional subspace $\operatorname{ker}(\mathrm{id}-\tilde{\sigma})$. Then $n$ is a vector normal to $\operatorname{ran}(\mathrm{id}-\sigma)$. The preimage (id $-\sigma)^{-1}(\xi) \subset V$ is an affine line in the direction of $n$. Since $\xi \in V_{\sigma}$, this line intersects $A$; hence it intersects the boundary $\partial \bar{A}$ in exactly two points $x$ and $x^{\prime}$. By (1), $x$ and $x^{\prime}$ are contained in two distinct codimension 1 boundary faces $A_{i}$ and $A_{i^{\prime}}$. Since $n$ is inward-pointing at one of the boundary faces, and outward-pointing at the other, the inner products $\left\langle n, \alpha_{i}\right\rangle$ and $\left\langle n, \alpha_{i^{\prime}}\right\rangle$ are both nonzero, with opposite signs. Let $w=\sigma s_{i}$ and let $w^{\prime}=\sigma s_{i^{\prime}}$. We will show that $w \in W_{\text {reg }}^{\text {aff }}$, that is, $\tilde{w} \in W_{\text {reg }}$ (the proof for $w^{\prime}$ is 


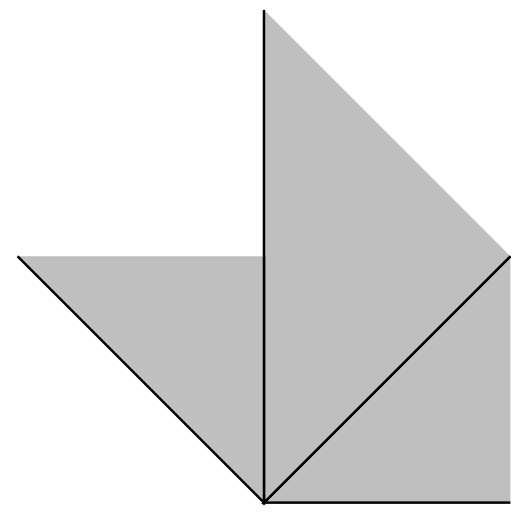

Figure 1. The set $X$ for the root system $\mathbf{B}_{2}$.

similar). Let $z \in V$ with $\tilde{w} z=z$. Then $\tilde{\sigma}^{-1} z=\tilde{s}_{i} z$, so

$$
\left(\mathrm{id}-\tilde{\sigma}^{-1}\right)(z)=\left(\mathrm{id}-\tilde{s}_{i}\right)(z)=\left\langle\alpha_{i}, z\right\rangle \alpha_{i}^{\vee} .
$$

The left side lies in $\operatorname{ran}(\mathrm{id}-\tilde{\sigma})$, which is orthogonal to $n$, while the right side is proportional to $\alpha_{i}$. Since $\left\langle n, \alpha_{i}\right\rangle \neq 0$, this is only possible if both sides are 0 . Thus $z$ is fixed under $\tilde{\sigma}$, and hence a multiple of $n$. On the other hand we have $\left\langle\alpha_{i}, z\right\rangle=0$; hence using again that $\left\langle n, \alpha_{i}\right\rangle \neq 0$ we obtain $z=0$. This shows $\operatorname{ker}(\mathrm{id}-\tilde{w})=0$.

As we had seen above, $n_{w, i}$ is a vector normal to $\operatorname{ran}(\mathrm{id}-\sigma)$ and hence is a multiple of $n$. By Lemma 3.2, it is a positive multiple if and only if $\left\langle n, \alpha_{i}\right\rangle>0$. But then $\left\langle n, \alpha_{i^{\prime}}\right\rangle<0$, and so $n_{w^{\prime}, i^{\prime}}$ is a negative multiple of $n$. This shows that $V_{w}$ and $V_{w^{\prime}}$ are on opposite sides of the hyperplane ran $(\mathrm{id}-\sigma)$.

Consider the union

$$
X:=\bigcup_{w \in W} V_{w}
$$

over $W \subset W^{\text {aff }}$. Thus $\bigcup_{w \in W^{\text {aff }}} V_{w}=\bigcup_{\lambda \in \Lambda}(\lambda+X)$. The statement of Theorem 1.2 means in particular that $X$ is a fundamental domain for the action of $\Lambda$. Figure 1 and Figure 2 give pictures of $X$ for the root systems $\mathbf{B}_{\mathbf{2}}$ and $\mathbf{G}_{\mathbf{2}}$. The shaded regions are the top-dimensional polytopes (that is, the sets $V_{w}$ for id $-w$ invertible), the dark lines are the 1-dimensional polytopes (corresponding to reflections), and the origin corresponds to $w=\mathrm{id}$.

Proposition 3.4. (a) The sets $\lambda+\operatorname{int}(\bar{X})$ for $\lambda \in \Lambda$ are disjoint, and

$$
\bigcup_{\lambda \in \Lambda} \lambda+\bar{X}=V .
$$




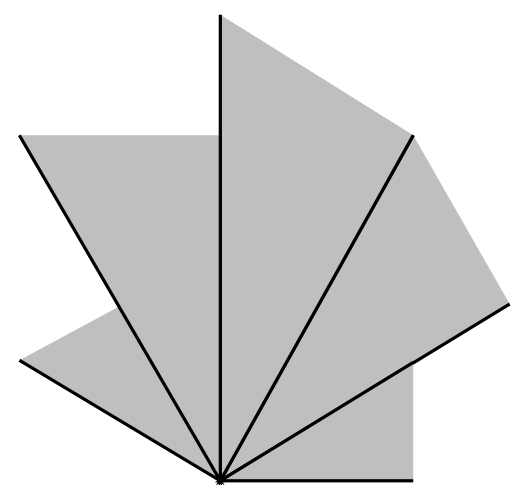

Figure 2. The set $X$ for the root system $\mathbf{G}_{2}$.

(b) The open polytopes $V_{w}$ for $w \in W_{\text {reg }}^{\text {aff }}$ are disjoint, and

$$
\bigcup_{w \in W_{\mathrm{reg}}^{\text {aff }}} \bar{V}_{w}=V .
$$

Proof. Since the collection of closed polytopes $\bar{V}_{w}$ for $w \in W_{\text {reg }}$ is locally finite, the union $V^{\prime}:=\bigcup_{w \in W_{\mathrm{reg}}}^{\text {aff }} \bar{V}_{w}$ is a closed polyhedral subset of $V$. Proposition 3.3 shows that a point $\xi \in V_{w, i}$ cannot contribute to the boundary of this subset unless it lies in $\bigcup_{\sigma \in W^{\text {aff }}} \bigcup_{|I| \geq 2} V_{\sigma, I}$. We therefore see that the boundary has codimension at least 2, and hence is empty since $V^{\prime}$ is a closed polyhedron. This proves $V^{\prime}=V$, and also $\bigcup_{\lambda \in \Lambda}(\lambda+\bar{X})=V$ with $X$ as defined in (2). Hence the volume $\operatorname{vol}(X)$ (for the Riemannian measure on $V$ defined by the inner product) must be at least the volume of a fundamental domain for the action of $\Lambda$ :

$$
\operatorname{vol}(X) \geq|W| \operatorname{vol}(A) .
$$

On the other hand, $\operatorname{vol}\left(V_{w}\right)=\operatorname{vol}((\mathrm{id}-w)(A))=\operatorname{det}(\mathrm{id}-w) \operatorname{vol}(A)$, so

$$
\operatorname{vol}(X) \leq \sum_{w \in W} \operatorname{vol}\left(V_{w}\right)=\operatorname{vol}(A) \sum_{w \in W} \operatorname{det}(\mathrm{id}-w)=|W| \operatorname{vol}(A),
$$

where we used that $\sum_{w \in W} \operatorname{det}(\mathrm{id}-w)=|W|$ from [Bourbaki 1975, page 134]. This confirms $\operatorname{vol}(X)=|W| \operatorname{vol}(A)$. It follows that the sets $\lambda+\operatorname{int}(\bar{X})$ are pairwise disjoint, or else the inequality (3) would be strict. Similarly that the sets $V_{w}$ for $w \in W_{\text {reg }}$ are disjoint, or else the inequality (4) would be strict. (Of course, this also follows from Waldspurger's Theorem 1.1 since $C_{w} \subset D_{w}$.) Hence all $V_{w}$ for $w \in W_{\text {reg }}^{\text {aff }}$ are disjoint.

To proceed, we quote the following result from Waldspurger's paper, where it is stated in greater generality . 
Proposition 3.5 [Waldspurger 2007, Lemme]. Given $w \in W$ and a proper subset $I \subset\{0, \ldots, l\}$, there exists a unique $q \in W_{I}$ such that

$$
\operatorname{ker}(\mathrm{id}-w q) \cap\left\{x \in V \mid\left\langle\alpha_{i}, x\right\rangle>0 \text { for all } i \in I\right\} \neq \varnothing .
$$

Following [Waldspurger 2007] we use this to prove,

Proposition 3.6. Every element of $V$ is contained in some $V_{w}$ for $w \in W^{\text {aff }}$ :

$$
\bigcup_{w \in W^{\text {aff }}} V_{w}=V .
$$

Proof. Let $\xi \in V$ be given. Pick $w \in W_{\text {reg }}^{\text {aff }}$ with $\xi \in \bar{V}_{w}$, and let $I \subset\{0, \ldots, l\}$ with $\xi \in V_{w, I}$. Then $x:=(\mathrm{id}-w)^{-1}(\xi) \in A_{I}$ is fixed under $W_{I}^{\text {aff }}$. By Proposition 3.5 we may choose $\tilde{q} \in W_{I}$ and $n \in V$ such that

(a) $\tilde{w} \tilde{q}(n)=n$,

(b) $\left\langle\alpha_{i}, n\right\rangle>0$ for all $i \in I$.

Taking $\|n\|$ sufficiently small, we have $x+n \in A$, and

$$
(\mathrm{id}-w q)(x+n)=(\mathrm{id}-w q)(x)+(\mathrm{id}-\tilde{w} \tilde{q}) n=(\mathrm{id}-w)(x)=\xi .
$$

This shows $\xi \in V_{w q}$.

\section{Disjointness of the sets $\lambda+X$}

To finish the proof of Theorem 1.2, we have to show that the union (5) is disjoint. Waldspurger's Theorem 1.1 shows that all $D_{w}=(\mathrm{id}-w)(C)$ for $w \in W$ are disjoint. (We refer to his paper for a very simple proof of this fact.) Hence the same is true of $V_{w} \subset D_{w}$ for $w \in W$. It remains to show that the sets $\lambda+X$ for $\lambda \in \Lambda$, with $X$ given by (2), are disjoint.

The closure $\bar{X}=\bigcup_{w \in W} \bar{V}_{w}$ only involves the top-dimensional polytopes:

Lemma 4.1. The closure of the set $X$ is the union $\bar{X}=\bigcup_{w \in W_{\mathrm{reg}}} \bar{V}_{w}$. Furthermore, $\operatorname{int}(\bar{X})=\operatorname{int}(X)$.

Proof. We must show that for any $\xi \in \bar{V}_{\sigma}$ with $\sigma \in W \backslash W_{\text {reg }}$, there exists a $w \in W_{\text {reg }}$ such that $\xi \in \bar{V}_{w}$. Using induction, it suffices to find $\sigma^{\prime} \in W$ such that $\xi \in \bar{V}_{\sigma^{\prime}}$ and $\operatorname{dim}\left(\operatorname{ker}\left(\mathrm{id}-\sigma^{\prime}\right)\right)=\operatorname{dim}(\operatorname{ker}(\mathrm{id}-\sigma))-1$. Let $\pi: V \rightarrow \operatorname{ker}(\mathrm{id}-\sigma)^{\perp}=\operatorname{ran}(\mathrm{id}-\sigma)$ denote the orthogonal projection. Then id $-\sigma$ restricts to an invertible transformation of $\pi(V)$, and $\bar{V}_{\sigma}$ is the image of $\pi(\bar{A})$ under this transformation. We have

$$
\pi(\bar{A})=\pi(\partial \bar{A})=\bigcup_{i=0}^{l} \pi\left(\bar{A}_{i}\right),
$$


and this continues to hold if we remove the index $i=0$ from the right side, as well as all indices $i$ for which $\operatorname{dim} \pi\left(A_{i}\right)<\operatorname{dim} \pi(V)$. That is, for each point $x \in \pi(\bar{A})$ there exists an index $i \neq 0$ such that $x \in \pi\left(\bar{A}_{i}\right)$, with $\operatorname{dim} \pi\left(A_{i}\right)=\operatorname{dim} \pi(V)$. Taking $x$ to be the preimage of $\xi$ under (id $-\sigma)\left.\right|_{\pi(V)}$, we have $\xi \in \bar{V}_{\sigma, i}$ with $i \neq 0$ and $\operatorname{dim} V_{\sigma, i}=$ $\operatorname{dim} \operatorname{ran}(\mathrm{id}-\sigma)$. Let $\sigma^{\prime}=\sigma s_{i} \in W$. Then $V_{\sigma, i}=V_{\sigma^{\prime}, i}$; hence $\operatorname{dim}\left(\operatorname{ran}\left(\mathrm{id}-\sigma^{\prime}\right)\right) \geq$ $\operatorname{dim} V_{\sigma, i}=\operatorname{dim}(\operatorname{ran}(\mathrm{id}-\sigma))$, which shows $\operatorname{dim} \operatorname{ker}\left(\mathrm{id}-\sigma^{\prime}\right) \leq \operatorname{dim} \operatorname{ker}(\mathrm{id}-\sigma)$. By elementary properties of reflection groups, the dimensions of the fixed point sets of $\sigma$ and $\sigma^{\prime}$ differ by either +1 or -1 . Hence $\operatorname{dim}\left(\operatorname{ker}\left(\mathrm{id}-\sigma^{\prime}\right)\right)=\operatorname{dim}(\operatorname{ker}(\mathrm{id}-\sigma))-1$, proving the first assertion of the lemma.

It follows in particular that the closure of $\operatorname{int}(\bar{X})$ equals that of $X$. Suppose $\xi \in \operatorname{int}(\bar{X})$. By Proposition 3.6 there exists $\lambda \in \Lambda$ with $\xi \in \lambda+X$. It follows that $\operatorname{int}(\bar{X})$ meets $\lambda+X$, and hence also meets $\lambda+\operatorname{int}(\bar{X})$. Since the $\Lambda$-translates of $\operatorname{int}(\bar{X})$ are pairwise disjoint (see Proposition 3.4), it follows that $\lambda=0$, that is, $\xi \in X$. This shows $\xi \in X \cap \operatorname{int}(\bar{X})=\operatorname{int}(X)$; hence $\operatorname{int}(\bar{X}) \subset \operatorname{int}(X)$. The opposite inclusion is obvious.

Since we already know that the sets $\lambda+\operatorname{int}(X)$ are disjoint, we are interested in $X \backslash \operatorname{int}(X) \subset \partial X=\bar{X} \backslash \operatorname{int}(X)$. Let us call a closed codimension 1 boundary face of the polyhedron $\bar{X}$ horizontal if its supporting hyperplane contains $V_{w, 0}$ for some $w \in W_{\text {reg }}$, and vertical if its supporting hyperplane contains $V_{w, i}$ for some $w \in W_{\text {reg }}$ and $i \neq 0$. These two cases are exclusive:

Lemma 4.2. Let $n$ be the inward-pointing normal vector to a codimension 1 face of $\bar{X}$. Then $\left\langle n, \alpha_{\max }\right\rangle \neq 0$. In fact, $\left\langle n, \alpha_{\max }\right\rangle<0$ for the horizontal faces and $\left\langle n, \alpha_{\max }\right\rangle>0$ for the vertical faces.

Proof. Given a codimension 1 boundary face of $\bar{X}$, pick any point $\xi$ in that boundary face not lying in $\bigcup_{w \in W^{\text {aff }}} \bigcup_{|I| \geq 2} V_{w, I}$. Let $w \in W_{\text {reg }}$ and $i \in\{0, \ldots, l\}$ such that $\xi \in V_{w, i}$ and $n_{w, i}$ is an inward-pointing normal vector. By Proposition 3.3, there is a unique $i^{\prime} \neq i$ such that $\xi \in V_{w^{\prime}, i^{\prime}}$, where $w^{\prime}=w s_{i} s_{i^{\prime}}$. Since $V_{w}$ and $V_{w^{\prime}}$ lie on opposite sides of the affine hyperplane spanned by $V_{w, i}$, and $\xi$ is a boundary point of $\bar{X}$, we have $w^{\prime} \notin W$. Thus one of $i$ and $i^{\prime}$ must be zero. If $i=0$ (so that the given boundary face is horizontal) we obtain $\left\langle n_{w, 0}, \alpha_{\max }\right\rangle=-\left\langle n_{w, 0}, \alpha_{0}\right\rangle<0$. If $i^{\prime}=0$ we similarly obtain $\left\langle n_{w^{\prime}, 0}, \alpha_{\max }\right\rangle<0$; hence $\left\langle n_{w, i}, \alpha_{\max }\right\rangle>0$.

Lemma 4.3. Let $\xi \in X \backslash \operatorname{int}(X)$. Then there exists a vertical boundary face of $\bar{X}$ containing $\xi$. Equivalently, the complement $\partial \bar{X} \backslash(X \backslash \operatorname{int}(X))$ is contained in the union of horizontal boundary faces.

Proof. The alcove $A$ is invariant under multiplication by any scalar in $(0,1)$. Hence, the same is true for the sets $V_{w}$ for $w \in W$, as well as for $X$ and $\operatorname{int}(X)$. Hence, if $\xi \in X \backslash \operatorname{int}(X)$ there exists $t_{0}>1$ such that $t \xi \in X \backslash \operatorname{int}(X)$ for $1 \leq t<t_{0}$. The closed codimension 1 boundary face containing this line segment is necessarily vertical, 
since a line through the origin intersects the affine hyperplane $\left\{x \mid\left\langle n_{w, 0}, x-\xi\right\rangle=0\right\}$ in at most one point.

Proposition 4.4. For any $\xi \in X$, there exists $\epsilon>0$ such that $\xi+s \alpha_{\max } \in \operatorname{int}(X)$ for $0<s<\epsilon$.

Proof. If $\xi \in \operatorname{int}(X)$ there is nothing to show; hence suppose $\xi \in X \backslash \operatorname{int}(X)$. Suppose first that $\xi$ is not in the union of horizontal boundary faces of $\bar{X}$. Then there exists an open neighborhood $U$ of $\xi$ such that $U \cap X=U \cap \bar{X}$. All boundary faces of $\bar{X}$ meeting $\xi$ are vertical, and their inward-pointing normal vectors $n$ all satisfy $\left\langle n, \alpha_{\max }\right\rangle>0$. Hence, $\xi+s \alpha_{\max } \in \operatorname{int}(U \cap \bar{X})=\operatorname{int}(U \cap X) \subset X$ for $s>0$ sufficiently small.

For the general case, suppose by way of contradiction that for all $\epsilon>0$, there is $s \in(0, \epsilon)$ with $\xi+s \alpha_{\max } \notin \operatorname{int}(X)$. Since $\xi$ is contained in some vertical boundary face, one can choose $t>1$ so that $\xi^{\prime}:=t \xi \in X \backslash \operatorname{int}(X)$, but $\xi^{\prime}$ is not in the closure of the union of horizontal boundary faces. Given $\epsilon>0$, pick $s \in(0, \epsilon)$ such that $\xi+(s / t) \alpha_{\max } \notin \operatorname{int}(X)$. Since $\operatorname{int}(X)$ is invariant under multiplication by scalars in $(0,1)$, the complement $V \backslash \operatorname{int}(X)$ is invariant under multiplication by scalars in $(1, \infty)$; hence we obtain $\xi^{\prime}+s \alpha_{\max } \notin \operatorname{int}(X)$. This contradicts what we have shown above, and completes the proof.

Proposition 4.5. The sets $\lambda+X$ for $\lambda \in \Lambda$ are disjoint.

Proof. Suppose $\xi \in(\lambda+X) \cap\left(\lambda^{\prime}+X\right)$. By Proposition 4.4, we can choose $s>0$ so that $\xi+s \alpha_{\max } \in(\lambda+\operatorname{int}(X)) \cap\left(\lambda^{\prime}+\operatorname{int}(X)\right)$. Since the $\Lambda$-translates of $\operatorname{int}(X)$ are disjoint, it follows that $\lambda=\lambda^{\prime}$.

This completes the proof of Theorem 1.2. We conclude with some remarks on the properties of the decomposition $V=\bigcup_{w \in W^{\text {aff }}} V_{w}$.

Remarks 4.6. (a) The group of symmetries $\tau$ of the extended Dynkin diagram (that is, the outer automorphisms of the corresponding affine Lie algebra) acts by symmetries of the decomposition $V=\bigcup_{w \in W^{\text {aff }}} V_{w}$, as follows. Identify the nodes of the extended Dynkin diagram with the simple affine reflections $s_{0}, \ldots, s_{l}$. Then $\tau$ extends to a group automorphism of $W^{\text {aff }}$, taking $s_{i}$ to $\tau\left(s_{i}\right)$. This automorphism is implemented by a unique Euclidean transformation $g: V \rightarrow V$, that is,

$$
g w g^{-1}=\tau(w) \quad \text { for all } w \in W^{\text {aff }} .
$$

Then $g$ preserves $A$, and consequently

$$
g V_{w}=g(\mathrm{id}-w)(A)=(\mathrm{id}-\tau(w))(A)=V_{\tau(w)} \quad \text { for } w \in W^{\text {aff }} .
$$

(b) It is immediate from the definition that $-w: V \rightarrow V, x \mapsto-w x$ takes $V_{w^{-1}}$ into $V_{w}$ :

$$
-w\left(V_{w^{-1}}\right)=V_{w}
$$


(c) For any positive root $\alpha$, let $s_{\alpha}$ be the corresponding reflection. Then

$$
\left(\mathrm{id}-s_{\alpha}\right)(\xi)=\langle\alpha, \xi\rangle \alpha^{\vee}
$$

where $\alpha^{\vee}$ is the coroot corresponding to $\alpha$. Hence $D_{s_{\alpha}}$ is the relative interior of the line segment from 0 to $\lambda \alpha^{\vee}$, where $\lambda$ is the maximum value of the linear functional $\xi \mapsto\langle\alpha, \xi\rangle$ on the closed alcove $\bar{A}$. This maximum is achieved at one of the vertices. Let $\varpi_{1}^{\vee}, \ldots, \varpi_{l}^{\vee}$ be the fundamental coweights, defined by $\left\langle\alpha_{i}, \varpi_{j}^{\vee}\right\rangle=\delta_{i j}$ for $i, j=1, \ldots, l$. Let $c_{i} \in \mathbb{N}$ be the coefficients of $\alpha_{\max }$ relative to the simple roots: $\alpha_{\max }=\sum_{i=1}^{l} c_{i} \alpha_{i}$. Then the nonzero vertices of $A$ are $\varpi_{i}^{\vee} / c_{i}$. Similarly let $a_{i} \in \mathbb{Z}_{\geq 0}$ be the coefficients of $\alpha$, so that $\alpha=\sum_{i=1}^{l} a_{i} \alpha_{i}$. Then the value of $\alpha$ at the $i$-th vertex of $\bar{A}$ is $a_{i} / c_{i}$, and $\lambda$ is the maximum of those values. There are two interesting cases: First, if $\alpha=\alpha_{\max }$, then all $a_{i} / c_{i}=1$, and $\alpha^{\vee}=\alpha$. That is, the open line segment from the origin to the highest root always appears in the decomposition. Second, if $\alpha=\alpha_{i}$, then $a_{i}=1$ while all other $a_{j}$ vanish. In this case, one obtains the open line segment from the origin to $\left(1 / c_{i}\right) \alpha_{i}^{\vee}$.

(d) Every $V_{w}$ contains a distinguished 'base point'. Indeed, let $\rho \in V$ be the halfsum of positive roots, and $h^{\vee}=1+\left\langle\alpha_{\max }, \rho\right\rangle$ the dual Coxeter number. Then $\rho / h^{\vee} \in A$, and consequently $\rho / h^{\vee}-w\left(\rho / h^{\vee}\right) \in V_{w}$.

\section{Proof of Theorem 1.3}

The proof is very similar to the proof of Proposition 3.4; hence we will be brief. Each $V_{w}^{(S)}=(S-w)(A)$ is the interior of a simplex in $V$, with codimension 1 faces $V_{w, i}^{(S)}=(S-w)\left(A_{i}\right)$. As in the proof of Lemma 3.1, we see that

$$
n_{w, i}^{(S)}=\left(S-\tilde{w}^{-1}\right)^{-1} \alpha_{i}
$$

is an inward-pointing normal vector to the face $V_{w, i}^{(S)}$. For $S=0$ this simplifies to $n_{w, i}^{(0)}=-w \alpha_{i}$. If $w^{\prime}=w s_{i}$, then $V_{w, i}^{(S)}=V_{w^{\prime}, i}^{(S)}$, so that $n_{w, i}^{(S)}$ and $n_{w^{\prime}, i}^{(S)}$ are proportional. Since $n_{w, i}^{(0)}=-n_{w^{\prime}, i}^{(0)}$, continuity implies that $n_{w, i}^{(S)}$ is a negative multiple of $n_{w^{\prime}, i}^{(S)}$. As a consequence, we see that $V_{w}^{(S)}$ and $V_{w^{\prime}}^{(S)}$ are on opposite sides of affine hyperplane supporting $V_{w, i}^{(S)}=V_{w^{\prime}, i}^{(S)}$. Arguing as in the proof of Proposition 3.4, this shows that

$$
\bigcup_{w \in W^{\text {aff }}} \bar{V}_{w}^{(S)}=V
$$


Letting $X^{(S)}=\bigcup_{w \in W} V_{w}^{(S)}$, it follows that $V=\bigcup_{\lambda \in \Lambda}\left(\lambda+\bar{X}^{(S)}\right)$. Therefore we have $\operatorname{vol}\left(X^{(S)}\right) \geq|W| \operatorname{vol}(A)$. But

$$
\begin{aligned}
\operatorname{vol}\left(X^{(S)}\right) & \leq \sum_{w \in W} \operatorname{vol}((S-w)(A)) \\
& =\operatorname{vol}(A) \sum_{w \in W}|\operatorname{det}(S-w)| \\
& =\operatorname{vol}(A) \sum_{w \in W} \operatorname{det}\left(\mathrm{id}-S w^{-1}\right)=|W| \operatorname{vol}(A)
\end{aligned}
$$

using [Bourbaki 1975, page 134]. It follows that $\operatorname{vol}\left(X^{(S)}\right)=|W| \operatorname{vol}(A)$, which implies (as in the proof of Proposition 3.4) that all $\operatorname{int}\left(\bar{V}_{w}^{(S)}\right)=V_{w}^{(S)}$ are disjoint. This completes the proof.

Remark 5.1. Theorem 1.3 and its proof go through for any $S$ in the component of 0 in the set $\{S \in \operatorname{End}(V) \mid \operatorname{det}(S-w) \neq 0$ for all $w \in W\}$. For instance, the fact that $\operatorname{det}\left(\mathrm{id}-S w^{-1}\right)>0$ follows by continuity from $S=0$. On the other hand, the result becomes false if, for example, $S$ is a positive matrix with $S>2 \mathrm{id}$, since then $\sum_{w \in W}|\operatorname{det}(S-w)|=\sum_{w \in W} \operatorname{det}(S-w)=\operatorname{det}(S)|W|$; see [Bourbaki 1975, page 134].

\section{Acknowledgments}

I would like to thank Bert Kostant for telling me about Waldspurger's result, and the referee for helpful comments.

\section{References}

[Bourbaki 1975] N. Bourbaki, Éléments de mathématique, Fasc. XXXVIII: Groupes et algèbres de Lie. Chapitre VII: Sous-algèbres de Cartan, éléments réguliers. Chapitre VIII: Algèbres de Lie semisimples déployées, Actualités Scientifiques et Industrielles 1364, Hermann, Paris, 1975. MR 56 \#12077 Zbl 0329.17002

[Waldspurger 2007] J.-L. Waldspurger, "Une remarque sur les systèmes de racines", J. Lie Theory 17:3 (2007), 597-603. MR 2008i:20051 Zbl 1133.20029

Received January 12, 2009. Revised February 4, 2009.

ECKHARD MEINRENKEN

UNIVERSITY OF TORONTO

DEPARTMENT OF MATHEMATICS

$40 \mathrm{St}$. GeOrge StreEt

TORONTO, ONTARIO M4S 2E4

CANADA

mein@math.toronto.edu 